\title{
Type Trait (Co)Variance Components for Five Dairy Breeds
}

\author{
G. R. Wiggans, ${ }^{1}$ N. Gengler, ${ }^{2}$ and J. R. Wright ${ }^{1}$ \\ ${ }^{1}$ Animal Improvement Programs Laboratory, Agricultural Research Service, \\ USDA, Beltsville, MD 20705-2350 \\ ${ }^{2}$ Animal Science Unit, Gembloux Agricultural University, B-5030 Gembloux, Belgium \\ and National Fund for Scientifique Research, B-1000 Brussels, Belgium
}

\section{ABSTRACT}

(Co)variance components were estimated for final score and 14 or 15 linear type traits for the Ayrshire, Brown Swiss, Guernsey, Jersey, and Milking Shorthorn breeds. Appraisals from 1995 or later were used. New estimates were calculated to accommodate changes in scoring of traits and because of a change from multiplicative to additive adjustment for age and lactation stage. The adjustment method was changed for better support of the adjustment for heterogeneous variance within iteration, which was implemented in 2002 . The largest changes in heritability were an increase of 0.10 for rump angle for Milking Shorthorns and a decrease of 0.11 for udder depth for Jerseys. The new estimates of (co)variance components should provide improved accuracy of type evaluations, particularly for traits that have had variance changes over time.

(Key words: type trait, variance component estimation)

\section{INTRODUCTION}

A multitrait model has been used by the Animal Improvement Programs Laboratory, ARS, USDA (Beltsville, MD), to calculate genetic evaluations for linear type traits of all breeds except Holstein since February 1998 (Gengler et al., 1999). The Holstein Association USA (Brattleboro, VT) calculates the Holstein type evaluations. The multitrait analysis used in the USDA evaluations was implemented using a canonical transformation with missing data (Ducrocq and Besbes, 1993). The required (co)variance matrices, which include all linear traits for each breed, were estimated as described by Gengler et al. (1997) in 1997.

Changes in the appraisal system for linear type traits have been implemented since 1997. Scoring of body depth was discontinued during 2002 for the Jersey

Received November 20, 2003.

Accepted March 28, 2004.

Corresponding author: G. R. Wiggans; e-mail: wiggans@aipl. arsusda.gov.
Table 1. Criteria for selecting data.

\begin{tabular}{lll}
\hline Breed & $\begin{array}{l}\text { Minimum number of } \\
\text { cows scored per herd } \\
\text { appraisal date }\end{array}$ & $\begin{array}{l}\text { Minimum number of } \\
\text { times herd scored } \\
1995-2002\end{array}$ \\
\hline Ayrshire & 5 & No minimum \\
Brown Swiss & 10 & 4 \\
Guernsey $^{\text {Jersey }}{ }^{1}$ & 10 & 6 \\
Milking Shorthorn $^{\text {Tilking }}$ & 5 & 6 \\
\hline
\end{tabular}

${ }^{1}$ Additional edit of $10 \%$ sample based on herd code imposed.

breed. The Ayrshire breed now assigns only nine different scores, but these range over a 50-point scale for compatibility with earlier data. Beginning in 1994, a substantial increase in within-herd-year variance occurred in the Brown Swiss breed.

An adjustment for heterogeneous variance was implemented in May 2001 for Jerseys and in August 2002 for the other breeds evaluated by USDA (Gengler et al., 2001a,b,c). The method of accounting for age and stage effects was simplified by discontinuing the multiplicative adjustment and by removing these effects from the model. They were removed from the model to exclude them from the heterogeneous variance adjustment. An additive adjustment was applied prior to iteration. The changes in data and model motivated the update of (co)variance estimates.

\section{MATERIALS AND METHODS}

\section{Data}

Based on an investigation of changes in variances within herd-year of appraisal, only appraisals from

Table 2. Numbers of herds, cows, and appraisals.

\begin{tabular}{lccc}
\hline Breed & Herds & Cows & Appraisals \\
\hline Ayrshire & 494 & 8854 & 12,049 \\
Brown Swiss & 183 & 21,560 & 37,902 \\
Guernsey & 113 & 16,332 & 31,311 \\
Jersey $^{1}$ & 45 & 23,811 & 38,999 \\
Milking Shorthorn $^{1}$ & 211 & 3,762 & 4,943 \\
\hline
\end{tabular}

${ }^{1}$ Data were selected from 356,357 appraisal records on a herd basis. 
Table 3. Number of levels of effects by breed.

\begin{tabular}{|c|c|c|c|c|c|}
\hline \multirow[b]{3}{*}{ Breed } & \multicolumn{3}{|c|}{ Fixed effects } & \multirow{2}{*}{\multicolumn{2}{|c|}{ Random effects }} \\
\hline & \multirow{2}{*}{$\begin{array}{l}\text { Herd } \\
\text { appraisal } \\
\text { date }\end{array}$} & \multirow[b]{2}{*}{ Age } & \multirow[b]{2}{*}{ Stage } & & \\
\hline & & & & $\begin{array}{l}\text { Permanent } \\
\text { environment }\end{array}$ & Animal \\
\hline Ayrshire & 979 & 27 & 22 & 5332 & 15,931 \\
\hline Brown Swiss & 2758 & 35 & 24 & 18,623 & 36,870 \\
\hline Guernsey & 2291 & 35 & 21 & 15,528 & 32,746 \\
\hline Jersey & 794 & 22 & 14 & 17,214 & 36,690 \\
\hline $\begin{array}{l}\text { Milking } \\
\text { Shorthorn }\end{array}$ & 462 & 28 & 24 & 3172 & 8383 \\
\hline
\end{tabular}

1995 and later were included. The amount of data was reduced to the capacity of the variance component estimation program by using the edits in Table 1 . These edits were intended to eliminate herds and appraisal dates with little information. The additional reduction required for the Jersey data was accomplished by selecting a $10 \%$ sample based on herd. Estimates from the sample were confirmed by calculating estimates from 3 additional samples. Numbers of herds, cows, and appraisals included for each breed are in Table 2.

\section{Estimation of Variance Components}

The variance component estimation program was provided by I. Misztal (University of Georgia, Athens), and calculations were as described by Gengler et al. (1997), using expectation-maximization REML and canonical transformation. Final score was not included in the multitrait analysis because the Ayrshire and Jersey breeds compute this trait from linear scores. Variance components for final score were estimated as a single trait. The model included fixed effects of herd appraisal date, age, and lactation stage and random effects of animal and permanent environment. The numbers of levels of effects by breed are in Table 3 . The
Table 4. Estimated heritability, repeatability, and total variance for final score by breed.

\begin{tabular}{lllc}
\hline Breed & Heritability & Repeatability & $\begin{array}{l}\text { Total } \\
\text { variance }\end{array}$ \\
\hline Ayrshire & 0.27 & 0.49 & 9.2 \\
Brown Swiss & 0.29 & 0.60 & 7.9 \\
Guernsey & 0.20 & 0.48 & 26.4 \\
Jersey & 0.19 & 0.44 & 32.4 \\
Milking Shorthorn & 0.20 & 0.60 & 9.1 \\
\hline
\end{tabular}

stage effect consisted of up to 8 levels within parity. Age was by 2-mo groups within parity except that larger numbers of months were included for the youngest and oldest ages. Following the policy for genetic evaluations, appraisals from only the first 2 parities were included for Jerseys.

The effects of the changes in (co)variance components were investigated by calculating correlations between evaluations using the old and new (co)variances. The change in variance of evaluations also was computed. Bulls used in AI, born in 1975 or later, and with a final score reliability of 75\% or more and cows born in 1995 or later were included.

\section{RESULTS}

\section{Heritabilities}

Heritabilities for final score by breed are given in Table 4. They ranged from 0.19 for Jersey to 0.29 for Brown Swiss. There was substantial variation in total variance. In 2002, 66\% of first-parity Ayrshires scored between 81 and 85 , whereas only $47 \%$ of Jerseys scored between 78 and 82 . These 2 ranges include the 5 most common scores for each breed. The total variances for the linear traits by breed are in Table 5 . The heritabilities and genetic and phenotypic correlations for the lin-

Table 5. Estimated total variance for 15 linear type traits by breed.

\begin{tabular}{llllll}
\hline Trait & & & & & Milking \\
Syrshire & Brown Swiss & Guernsey & Jersey & Shorthorn \\
\hline Stature & 58.6 & 41.3 & 56.5 & 30.4 & 33.1 \\
Strength & 37.2 & 33.1 & 37.8 & 29.5 & 29.2 \\
Dairy form & 42.6 & 42.0 & 46.8 & 37.8 & 39.2 \\
Foot angle & 46.2 & 41.0 & 37.8 & 35.2 & 25.6 \\
Rear legs (side view) & 34.3 & 30.5 & 34.1 & 30.1 & 21.1 \\
Body depth & 31.4 & 33.5 & 38.2 & - & 30.7 \\
Rump angle & 30.4 & 36.2 & 45.7 & 33.6 & 29.8 \\
Rump width & 38.6 & 25.2 & 31.2 & 25.6 & 22.1 \\
Fore udder attachment & 48.2 & 49.5 & 56.4 & 43.7 & 43.6 \\
Rear udder height & 43.2 & 42.1 & 47.2 & 44.6 & 40.1 \\
Rear udder width & 38.9 & 42.2 & 47.3 & 39.8 & 40.7 \\
Udder depth & 39.6 & 36.6 & 46.9 & 44.7 & 37.0 \\
Udder cleft & 44.1 & 47.8 & 45.4 & 29.5 & 32.0 \\
Front teat placement & 46.7 & 41.7 & 40.5 & 39.8 & 36.0 \\
Teat length & 43.2 & 45.0 & 35.8 & 29.8 & 35.1 \\
\hline
\end{tabular}




\begin{tabular}{|c|c|c|c|c|c|c|c|c|c|c|c|c|c|c|c|}
\hline Item & Stature & Strength & $\begin{array}{l}\text { Dairy } \\
\text { form }\end{array}$ & $\begin{array}{l}\text { Foot } \\
\text { angle }\end{array}$ & $\begin{array}{l}\text { Rear } \\
\text { legs } \\
\text { (side } \\
\text { view) }\end{array}$ & $\begin{array}{l}\text { Body } \\
\text { depth }\end{array}$ & $\begin{array}{l}\text { Rump } \\
\text { angle }\end{array}$ & $\begin{array}{l}\text { Rump } \\
\text { width }\end{array}$ & $\begin{array}{l}\text { Fore } \\
\text { udder } \\
\text { attachment }\end{array}$ & $\begin{array}{l}\text { Rear } \\
\text { udder } \\
\text { height }\end{array}$ & $\begin{array}{l}\text { Rear } \\
\text { udder } \\
\text { width }\end{array}$ & $\begin{array}{l}\text { Udder } \\
\text { depth }\end{array}$ & $\begin{array}{l}\text { Udder } \\
\text { cleft }\end{array}$ & $\begin{array}{l}\text { Front } \\
\text { teat } \\
\text { placement }\end{array}$ & $\begin{array}{l}\text { Teat } \\
\text { length }\end{array}$ \\
\hline Stature & 0.54 & 0.70 & 0.23 & 0.43 & 0.04 & 0.60 & -0.09 & 0.71 & 0.28 & 0.14 & 0.26 & 0.28 & 0.05 & 0.10 & 0.32 \\
\hline Strength & 0.44 & 0.31 & -0.17 & 0.46 & -0.20 & 0.81 & -0.13 & 0.71 & 0.21 & 0.04 & 0.34 & 0.01 & -0.11 & 0.00 & 0.26 \\
\hline Dairy form & 0.12 & -0.19 & 0.20 & 0.08 & 0.32 & 0.28 & -0.20 & 0.02 & -0.05 & 0.47 & 0.26 & -0.11 & 0.21 & 0.20 & 0.00 \\
\hline Foot angle & 0.17 & 0.23 & 0.00 & 0.15 & -0.45 & 0.38 & -0.20 & 0.56 & 0.36 & 0.32 & 0.47 & 0.24 & 0.15 & 0.20 & 0.22 \\
\hline Rear legs (side view) & -0.02 & -0.11 & 0.12 & -0.29 & 0.15 & -0.07 & -0.16 & -0.12 & -0.05 & -0.20 & -0.25 & 0.00 & -0.24 & 0.10 & -0.17 \\
\hline Body depth & 0.36 & 0.56 & 0.17 & 0.15 & -0.05 & 0.29 & -0.16 & 0.54 & 0.04 & 0.19 & 0.37 & -0.20 & 0.00 & 0.06 & 0.26 \\
\hline Rump angle & 0.01 & -0.04 & -0.05 & -0.08 & -0.04 & -0.03 & 0.28 & -0.18 & -0.33 & -0.23 & -0.19 & -0.19 & -0.10 & -0.05 & -0.07 \\
\hline Rump width & 0.46 & 0.52 & -0.02 & 0.23 & -0.08 & 0.37 & -0.07 & 0.35 & 0.26 & 0.21 & 0.38 & 0.16 & -0.04 & 0.07 & 0.29 \\
\hline Fore udder attachment & 0.13 & 0.11 & 0.05 & 0.17 & -0.04 & 0.07 & -0.16 & 0.15 & 0.21 & 0.43 & 0.56 & 0.61 & 0.27 & 0.54 & -0.01 \\
\hline Rear udder height & 0.08 & 0.01 & 0.32 & 0.12 & -0.05 & 0.11 & -0.12 & 0.10 & 0.28 & 0.26 & 0.75 & 0.16 & 0.49 & 0.25 & 0.03 \\
\hline Rear udder width & 0.15 & 0.19 & 0.23 & 0.16 & -0.09 & 0.19 & -0.08 & 0.24 & 0.26 & 0.53 & 0.19 & 0.16 & 0.40 & 0.44 & 0.13 \\
\hline Udder depth & 0.15 & -0.04 & -0.08 & 0.09 & -0.01 & -0.17 & -0.10 & 0.06 & 0.42 & 0.11 & 0.04 & 0.31 & 0.31 & 0.33 & -0.03 \\
\hline Udder cleft & 0.02 & 0.00 & 0.15 & 0.07 & -0.02 & 0.04 & -0.06 & 0.01 & 0.23 & 0.31 & 0.25 & 0.27 & 0.23 & 0.48 & -0.02 \\
\hline Front teat placement & 0.06 & 0.03 & 0.10 & 0.07 & 0.02 & 0.05 & -0.04 & 0.06 & 0.34 & 0.19 & 0.19 & 0.24 & 0.36 & 0.24 & -0.24 \\
\hline Teat length & 0.16 & 0.15 & 0.02 & 0.07 & -0.04 & 0.12 & -0.02 & 0.15 & -0.01 & 0.01 & 0.06 & -0.07 & -0.04 & -0.18 & 0.30 \\
\hline
\end{tabular}

gth

0.16

Table 7. Estimated genetic (above diagonal) and phenotypic (below diagonal) correlations and heritabilities (on diagonal) among 15 linear type traits of Brown Swiss.

\begin{tabular}{|c|c|c|c|c|c|c|c|c|c|c|c|c|c|c|c|}
\hline Item & Stature & Strength & $\begin{array}{l}\text { Dairy } \\
\text { form }\end{array}$ & $\begin{array}{l}\text { Foot } \\
\text { angle }\end{array}$ & $\begin{array}{l}\text { Rear } \\
\text { legs } \\
\text { (side } \\
\text { view) }\end{array}$ & $\begin{array}{l}\text { Body } \\
\text { depth }\end{array}$ & $\begin{array}{l}\text { Rump } \\
\text { angle }\end{array}$ & $\begin{array}{l}\text { Rump } \\
\text { width }\end{array}$ & $\begin{array}{l}\text { Fore } \\
\text { udder } \\
\text { attachment }\end{array}$ & $\begin{array}{l}\text { Rear } \\
\text { udder height }\end{array}$ & $\begin{array}{l}\text { Rear } \\
\text { udder } \\
\text { width }\end{array}$ & $\begin{array}{l}\text { Udder } \\
\text { depth }\end{array}$ & $\begin{array}{l}\text { Udder } \\
\text { cleft }\end{array}$ & $\begin{array}{l}\text { Front } \\
\text { teat } \\
\text { placement }\end{array}$ & $\begin{array}{l}\text { Teat } \\
\text { length }\end{array}$ \\
\hline Stature & 0.43 & 0.64 & 0.44 & 0.32 & 0.00 & 0.54 & 0.13 & 0.66 & 0.13 & 0.25 & 0.25 & 0.19 & 0.12 & 0.08 & 0.20 \\
\hline Strength & 0.44 & 0.20 & 0.10 & 0.29 & -0.04 & 0.85 & 0.03 & 0.85 & 0.10 & 0.03 & 0.35 & -0.14 & 0.12 & 0.08 & 0.20 \\
\hline Dairy form & 0.19 & -0.11 & 0.18 & 0.02 & 0.15 & 0.44 & -0.11 & 0.15 & 0.03 & 0.52 & 0.51 & -0.11 & 0.20 & 0.19 & 0.03 \\
\hline Foot angle & 0.13 & 0.13 & 0.00 & 0.13 & -0.40 & 0.17 & -0.07 & 0.27 & 0.25 & 0.17 & 0.17 & 0.18 & 0.13 & 0.13 & -0.01 \\
\hline Rear legs (side view) & -0.02 & -0.07 & 0.10 & -0.19 & 0.18 & 0.03 & 0.00 & 0.00 & -0.13 & -0.08 & -0.04 & -0.10 & -0.06 & -0.04 & 0.02 \\
\hline Body depth & 0.38 & 0.64 & 0.19 & 0.09 & -0.01 & 0.25 & -0.01 & 0.66 & -0.01 & 0.13 & 0.42 & -0.31 & 0.18 & 0.13 & 0.16 \\
\hline Rump angle & 0.11 & 0.00 & -0.02 & -0.03 & 0.01 & 0.00 & 0.27 & 0.02 & -0.25 & -0.24 & -0.15 & -0.16 & -0.07 & -0.10 & 0.01 \\
\hline Rump width & 0.39 & 0.51 & -0.01 & 0.11 & -0.02 & 0.43 & 0.02 & 0.18 & 0.14 & 0.13 & 0.40 & -0.03 & 0.17 & 0.09 & 0.15 \\
\hline Fore udder attachment & 0.08 & 0.16 & -0.02 & 0.13 & -0.08 & 0.10 & -0.13 & 0.11 & 0.22 & 0.57 & 0.36 & 0.73 & 0.24 & 0.43 & -0.13 \\
\hline Rear udder height & 0.11 & 0.01 & 0.32 & 0.10 & -0.05 & 0.08 & -0.10 & 0.07 & 0.31 & 0.22 & 0.67 & 0.36 & 0.35 & 0.29 & 0.01 \\
\hline Rear udder width & 0.13 & 0.18 & 0.31 & 0.11 & -0.06 & 0.24 & -0.05 & 0.21 & 0.21 & 0.52 & 0.19 & -0.08 & 0.37 & 0.32 & 0.00 \\
\hline Udder depth & 0.09 & -0.06 & -0.13 & 0.06 & -0.04 & -0.17 & -0.09 & -0.03 & 0.41 & 0.14 & -0.12 & 0.34 & 0.22 & 0.32 & -0.17 \\
\hline Udder cleft & 0.05 & 0.05 & 0.09 & 0.05 & -0.01 & 0.08 & -0.04 & 0.06 & 0.16 & 0.19 & 0.19 & 0.19 & 0.22 & 0.45 & -0.05 \\
\hline Front teat placement & 0.04 & 0.07 & 0.03 & 0.05 & -0.01 & 0.08 & -0.05 & 0.04 & 0.27 & 0.13 & 0.12 & 0.24 & 0.29 & 0.27 & -0.37 \\
\hline Teat length & 0.12 & 0.09 & 0.04 & 0.01 & 0.00 & 0.10 & 0.01 & 0.08 & -0.06 & 0.02 & 0.05 & -0.12 & 0.01 & -0.19 & 0.34 \\
\hline
\end{tabular}


Table 8. Estimated genetic (above diagonal) and phenotypic (below diagonal) correlations and heritabilities (on diagonal) among 15 linear type traits of Guernseys.

\begin{tabular}{|c|c|c|c|c|c|c|c|c|c|c|c|c|c|c|c|}
\hline Item & Stature & Strength & $\begin{array}{l}\text { Dairy } \\
\text { form }\end{array}$ & $\begin{array}{l}\text { Foot } \\
\text { angle }\end{array}$ & $\begin{array}{l}\text { Rear } \\
\text { legs } \\
\text { (side } \\
\text { view) }\end{array}$ & $\begin{array}{l}\text { Body } \\
\text { depth }\end{array}$ & $\begin{array}{l}\text { Rump } \\
\text { angle }\end{array}$ & $\begin{array}{l}\text { Rump } \\
\text { width }\end{array}$ & $\begin{array}{l}\text { Fore } \\
\text { udder } \\
\text { attachment }\end{array}$ & $\begin{array}{l}\text { Rear } \\
\text { udder } \\
\text { height }\end{array}$ & $\begin{array}{l}\text { Rear } \\
\text { udder } \\
\text { width }\end{array}$ & $\begin{array}{l}\text { Udder } \\
\text { depth }\end{array}$ & $\begin{array}{l}\text { Udder } \\
\text { cleft }\end{array}$ & $\begin{array}{l}\text { Front } \\
\text { teat } \\
\text { placement }\end{array}$ & $\begin{array}{l}\text { Teat } \\
\text { length }\end{array}$ \\
\hline Stature & 0.49 & 0.68 & 0.63 & 0.30 & 0.03 & 0.63 & 0.26 & 0.73 & -0.11 & 0.32 & 0.47 & -0.15 & 0.11 & 0.01 & 0.32 \\
\hline Strength & 0.45 & 0.22 & 0.36 & 0.26 & 0.07 & 0.82 & 0.08 & 0.81 & 0.01 & 0.16 & 0.45 & -0.22 & 0.16 & 0.07 & 0.37 \\
\hline Dairy form & 0.36 & 0.07 & 0.28 & 0.20 & -0.06 & 0.63 & 0.06 & 0.47 & -0.23 & 0.51 & 0.71 & -0.42 & 0.22 & 0.07 & 0.18 \\
\hline Foot angle & 0.13 & 0.15 & 0.05 & 0.10 & -0.29 & 0.16 & -0.06 & 0.30 & 0.10 & 0.31 & 0.29 & 0.05 & 0.18 & 0.07 & 0.12 \\
\hline Rear legs (side view) & -0.03 & -0.08 & 0.03 & -0.18 & 0.16 & 0.06 & -0.08 & 0.07 & -0.06 & -0.27 & -0.22 & -0.03 & -0.23 & -0.06 & -0.04 \\
\hline Body depth & 0.46 & 0.60 & 0.37 & 0.10 & -0.03 & 0.32 & 0.11 & 0.67 & -0.11 & 0.23 & 0.50 & -0.37 & 0.22 & 0.12 & 0.30 \\
\hline Rump angle & 0.18 & 0.01 & 0.05 & -0.04 & -0.01 & 0.04 & 0.41 & 0.05 & -0.22 & -0.05 & -0.02 & -0.18 & -0.08 & -0.04 & 0.03 \\
\hline Rump width & 0.48 & 0.53 & 0.19 & 0.13 & -0.02 & 0.45 & 0.03 & 0.29 & 0.00 & 0.32 & 0.54 & -0.20 & 0.18 & 0.06 & 0.39 \\
\hline Fore udder attachment & -0.03 & 0.11 & -0.11 & 0.09 & -0.08 & 0.01 & -0.15 & 0.05 & 0.29 & 0.40 & 0.06 & 0.80 & 0.19 & 0.51 & -0.14 \\
\hline Rear udder height & 0.20 & 0.09 & 0.33 & 0.11 & -0.11 & 0.15 & -0.03 & 0.17 & 0.29 & 0.28 & 0.72 & 0.21 & 0.36 & 0.29 & 0.04 \\
\hline Rear udder width & 0.28 & 0.26 & 0.43 & 0.14 & -0.12 & 0.30 & 0.00 & 0.33 & 0.13 & 0.53 & 0.28 & -0.25 & 0.40 & 0.20 & 0.17 \\
\hline Udder depth & -0.07 & -0.06 & -0.23 & 0.05 & -0.04 & -0.19 & -0.13 & -0.08 & 0.53 & 0.16 & -0.10 & 0.40 & 0.18 & 0.36 & -0.27 \\
\hline Udder cleft & 0.05 & 0.07 & 0.14 & 0.06 & -0.05 & 0.11 & -0.05 & 0.08 & 0.17 & 0.24 & 0.28 & 0.18 & 0.21 & 0.37 & -0.01 \\
\hline Front teat placement & 0.02 & 0.07 & 0.03 & 0.04 & -0.02 & 0.08 & -0.04 & 0.05 & 0.36 & 0.19 & 0.15 & 0.29 & 0.28 & 0.31 & -0.29 \\
\hline Teat length & 0.20 & 0.16 & 0.11 & 0.03 & -0.01 & 0.16 & 0.02 & 0.18 & -0.05 & 0.04 & 0.12 & -0.15 & 0.04 & -0.12 & 0.34 \\
\hline
\end{tabular}

Table 9. Estimated genetic (above diagonal) and phenotypic (below diagonal) correlations and heritabilities (on diagonal) among 14 linear type traits of Jerseys.

\begin{tabular}{|c|c|c|c|c|c|c|c|c|c|c|c|c|c|c|}
\hline Item & Stature & Strength & $\begin{array}{l}\text { Dairy } \\
\text { form }\end{array}$ & $\begin{array}{l}\text { Foot } \\
\text { angle }\end{array}$ & $\begin{array}{l}\text { Rear } \\
\text { legs } \\
\text { (side view) }\end{array}$ & $\begin{array}{l}\text { Rump } \\
\text { angle }\end{array}$ & $\begin{array}{l}\text { Rump } \\
\text { width }\end{array}$ & $\begin{array}{l}\text { Fore } \\
\text { udder } \\
\text { attachment }\end{array}$ & $\begin{array}{l}\text { Rear } \\
\text { udder } \\
\text { height }\end{array}$ & $\begin{array}{l}\text { Rear } \\
\text { udder } \\
\text { width }\end{array}$ & $\begin{array}{l}\text { Udder } \\
\text { depth }\end{array}$ & $\begin{array}{l}\text { Udder } \\
\text { cleft }\end{array}$ & $\begin{array}{l}\text { Front } \\
\text { teat } \\
\text { placement }\end{array}$ & $\begin{array}{l}\text { Teat } \\
\text { length }\end{array}$ \\
\hline Stature & 0.37 & 0.80 & 0.40 & 0.40 & 0.00 & 0.29 & 0.77 & 0.21 & 0.24 & 0.36 & 0.16 & 0.16 & 0.15 & 0.30 \\
\hline Strength & 0.55 & 0.21 & 0.32 & 0.43 & -0.11 & 0.23 & 0.89 & 0.20 & 0.18 & 0.42 & -0.04 & 0.25 & 0.25 & 0.33 \\
\hline Dairy form & 0.26 & 0.25 & 0.21 & 0.29 & 0.20 & 0.09 & 0.46 & 0.32 & 0.68 & 0.86 & -0.09 & 0.44 & 0.47 & 0.13 \\
\hline Foot angle & 0.19 & 0.22 & 0.19 & 0.11 & -0.49 & -0.05 & 0.47 & 0.40 & 0.34 & 0.37 & 0.27 & 0.27 & 0.24 & 0.16 \\
\hline Rear legs (side view) & -0.02 & -0.05 & 0.07 & -0.22 & 0.07 & 0.07 & 0.01 & -0.08 & 0.05 & 0.10 & -0.12 & 0.16 & 0.15 & -0.13 \\
\hline Rump angle & 0.17 & 0.13 & 0.09 & 0.00 & 0.03 & 0.22 & 0.18 & -0.09 & -0.01 & 0.03 & -0.09 & 0.01 & 0.01 & 0.08 \\
\hline Rump width & 0.45 & 0.57 & 0.29 & 0.24 & -0.03 & 0.12 & 0.18 & 0.27 & 0.32 & 0.53 & 0.01 & 0.29 & 0.31 & 0.27 \\
\hline Fore udder attachment & 0.12 & 0.16 & 0.23 & 0.23 & -0.03 & -0.05 & 0.18 & 0.19 & 0.77 & 0.62 & 0.76 & 0.45 & 0.61 & 0.07 \\
\hline Rear udder height & 0.17 & 0.17 & 0.53 & 0.22 & 0.00 & 0.02 & 0.21 & 0.57 & 0.26 & 0.86 & 0.45 & 0.56 & 0.51 & 0.13 \\
\hline Rear udder width & 0.24 & 0.31 & 0.63 & 0.23 & 0.01 & 0.05 & 0.34 & 0.42 & 0.72 & 0.22 & 0.14 & 0.59 & 0.62 & 0.15 \\
\hline Udder depth & 0.05 & -0.06 & -0.05 & 0.13 & -0.04 & -0.06 & -0.02 & 0.48 & 0.33 & 0.10 & 0.27 & 0.29 & 0.33 & -0.04 \\
\hline Udder cleft & 0.09 & 0.14 & 0.31 & 0.16 & 0.06 & 0.02 & 0.16 & 0.31 & 0.42 & 0.42 & 0.22 & 0.17 & 0.68 & 0.16 \\
\hline Front teat placement & 0.10 & 0.16 & 0.29 & 0.15 & 0.04 & 0.01 & 0.18 & 0.39 & 0.39 & 0.39 & 0.25 & 0.45 & 0.20 & -0.01 \\
\hline Teat length & 0.18 & 0.20 & 0.14 & 0.09 & -0.01 & 0.04 & 0.18 & 0.05 & 0.11 & 0.15 & -0.04 & 0.14 & 0.06 & 0.19 \\
\hline
\end{tabular}


ear traits for each breed are given in Tables 6 through 10. The variance components can be derived from the total variance and the heritabilities and correlations. The highest heritability was 0.54 for stature in Ayrshires, and the lowest was 0.07 for rear legs (side view) in Jerseys. Changes in heritability between this analysis and those of Gengler et al. (1997) are given in Table 11. The largest increase was 0.10 for rump angle in Milking Shorthorns. The largest decrease was 0.11 for udder depth in Jerseys. Most changes had an absolute value of 0.03 or less. The other Jersey samples gave results similar to the sample described in Table 2.

\section{Correlations}

The largest genetic correlation was 0.84 between rear udder width and rear udder height in Milking Shorthorns. The most negative correlation was -0.49 between rear legs (side view) and foot angle in Jerseys. Phenotypic correlations generally followed similar patterns but with smaller magnitude.

\section{Effect on Evaluations}

Correlations between evaluations using the old and new (co)variances ranged from 0.924 to 0.999 for bulls and 0.819 to 0.997 for cows. Final score and stature had the highest correlations across breed. This was expected because final score was evaluated as a single trait, therefore changes in covariances had no effect. Because stature has a high heritability, other traits add relatively little information to evaluation of stature. Changes in covariances of stature with other traits, therefore, had little effect. Correlations with rear legs (side view) were among the lowest. This trait has a low heritability; therefore changes in correlations with other traits can have a large effect.

The changes in variances in Tables 12 and 13 reflect the changes in Table 11. For Ayrshires, heritabilities were generally reduced, and consequently the variances of evaluations also declined. For Brown Swiss, the reverse occurred. Heritabilities increased as did variances of evaluations. Changes were generally slightly larger for cows than for bulls.

\section{DISCUSSION}

Changes in data over time suggest variance component estimates should be updated periodically either with base changes or major changes in the evaluation system. The heterogeneous variance adjustment does not force the variance of evaluations to be stable over time. The variance of evaluations is affected by the variance of the appraisals. A further step to stabilize

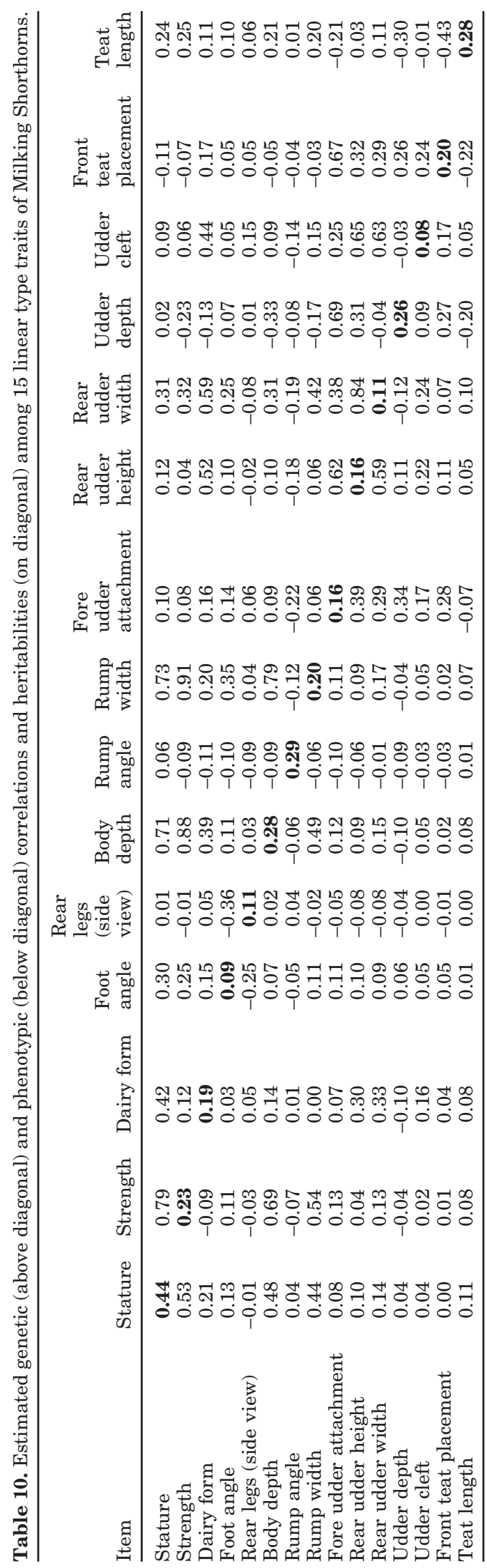


Table 11. Changes (new - old) in heritability estimates for type traits by breed.

\begin{tabular}{lclrrr}
\hline & & & & & \multicolumn{1}{c}{ Milking } \\
Trait & Ayrshire & Brown Swiss & Guernsey & Jersey & Shorthorn \\
\hline Final score & 0.007 & 0.016 & -0.059 & -0.035 & -0.080 \\
Stature & 0.082 & 0.029 & 0.008 & -0.025 & 0.024 \\
Strength & -0.008 & 0.004 & -0.071 & -0.036 & -0.026 \\
Dairy form & -0.041 & 0.006 & -0.060 & -0.022 & 0.006 \\
Foot angle & 0.044 & 0.043 & -0.018 & 0.001 & -0.001 \\
Rear legs (side view) & 0.058 & 0.024 & -0.026 & -0.034 & 0.021 \\
Body depth & -0.085 & 0.012 & -0.006 & - & -0.019 \\
Rump angle & -0.029 & 0.005 & 0.015 & -0.096 & 0.102 \\
Rump width & 0.061 & 0.024 & -0.010 & -0.021 & 0.043 \\
Fore udder attachment & -0.053 & 0.024 & 0.058 & -0.035 & 0.055 \\
Rear udder height & -0.030 & 0.010 & -0.035 & -0.006 & 0.031 \\
Rear udder width & -0.035 & 0.020 & -0.040 & -0.005 & -0.009 \\
Udder depth & 0.062 & 0.058 & 0.018 & -0.110 & -0.015 \\
Udder cleft & -0.022 & 0.062 & 0.031 & -0.030 & 0.030 \\
Front teat placement & -0.003 & 0.058 & 0.039 & -0.046 & 0.026 \\
Teat length & 0.009 & 0.028 & 0.037 & -0.072 & 0.043 \\
\hline
\end{tabular}

Table 12. Changes (new - old) in variances of type trait evaluations for cows born in 1995 or later by breed.

\begin{tabular}{|c|c|c|c|c|c|}
\hline Trait & Ayrshire & Brown Swiss & Guernsey & Jersey & $\begin{array}{l}\text { Milking } \\
\text { Shorthorn }\end{array}$ \\
\hline Final score & -0.05 & 0.01 & -0.16 & -0.09 & -0.05 \\
\hline Stature & -0.55 & 0.14 & -0.09 & -0.23 & 0.06 \\
\hline Strength & -0.30 & 0.01 & -0.29 & -0.12 & -0.14 \\
\hline Dairy form & -0.42 & 0.01 & -0.29 & -0.30 & -0.13 \\
\hline Foot angle & -0.11 & 0.06 & -0.04 & -0.06 & -0.01 \\
\hline Rear legs (side view) & 0.01 & 0.02 & -0.14 & -0.10 & 0.01 \\
\hline Body depth & -0.63 & 0.01 & -0.16 & - & -0.16 \\
\hline Rump angle & -0.61 & 0.05 & -0.01 & -0.35 & 0.15 \\
\hline Rump width & -0.34 & 0.03 & -0.08 & -0.08 & 0.05 \\
\hline Fore udder attachment & -0.62 & 0.11 & 0.04 & -0.43 & 0.06 \\
\hline Rear udder height & -0.79 & 0.05 & -0.20 & -0.33 & 0.02 \\
\hline Rear udder width & -0.49 & 0.04 & -0.10 & -0.18 & -0.11 \\
\hline Udder depth & -0.48 & 0.12 & 0.03 & -0.76 & -0.19 \\
\hline Udder cleft & -0.52 & 0.13 & 0.02 & -0.15 & 0.04 \\
\hline Front teat placement & -0.71 & 0.10 & -0.06 & -0.32 & -0.19 \\
\hline Teat length & -0.73 & 0.11 & -0.05 & -0.25 & -0.19 \\
\hline
\end{tabular}

Table 13. Changes (new - old) in variances of type trait evaluations for AI bulls born in 1975 or later and with a final score reliability of $75 \%$ or more by breed.

\begin{tabular}{|c|c|c|c|c|c|}
\hline Trait & Ayrshire & Brown Swiss & Guernsey & Jersey & $\begin{array}{l}\text { Milking } \\
\text { Shorthorn }\end{array}$ \\
\hline Final score & -0.11 & 0.02 & -0.21 & -0.12 & -0.06 \\
\hline Stature & -1.08 & 0.09 & -0.35 & -0.25 & 0.09 \\
\hline Strength & -0.55 & 0.00 & -0.39 & -0.13 & -0.21 \\
\hline Dairy form & -0.44 & 0.01 & -0.42 & -0.68 & -0.28 \\
\hline Foot angle & -0.19 & 0.05 & -0.04 & -0.11 & 0.00 \\
\hline Rear legs (side view) & -0.04 & 0.03 & -0.20 & -0.18 & 0.00 \\
\hline Body depth & -0.87 & 0.00 & -0.30 & - & -0.12 \\
\hline Rump angle & -0.89 & 0.04 & -0.10 & -0.33 & -0.03 \\
\hline Rump width & -0.63 & 0.03 & -0.14 & -0.12 & 0.10 \\
\hline Fore udder attachment & -1.26 & 0.07 & -0.11 & -0.44 & 0.04 \\
\hline Rear udder height & -1.22 & 0.06 & -0.29 & -0.50 & 0.05 \\
\hline Rear udder width & -0.72 & 0.04 & -0.25 & -0.32 & -0.13 \\
\hline Udder depth & -0.80 & 0.06 & -0.10 & -0.59 & -0.36 \\
\hline Udder cleft & -0.59 & 0.07 & -0.07 & -0.17 & 0.06 \\
\hline Front teat placement & -0.95 & 0.07 & -0.19 & -0.40 & -0.49 \\
\hline Teat length & -1.08 & 0.08 & -0.32 & -0.34 & -0.59 \\
\hline
\end{tabular}


variance of evaluations by specifying a base period for variance has been developed. This change is expected to add to the stability of evaluations and reduce the effects of changes in the variability of the scores assigned. The new variance components should improve the correspondence between the evaluations and the current characteristics of the appraisal data. Heritabilities were generally similar to those estimated from earlier data but with relatively large changes for a few traits. The heritabilities and genetic variances presented are posted on the Interbull website (http://www-interbull. slu.se/national_ges_info2/framesida-ges.htm), along with those from other countries for comparison.

\section{ACKNOWLEDGMENTS}

The authors thank I. Misztal, University of Georgia (Athens), for providing the computer program to estimate variance components and L. L. M. Thornton, Animal Improvement Programs Laboratory (Beltsville, $\mathrm{MD}$ ), for assistance in manuscript review. N. Gengler, who is a research associate of the National Fund for Scientific Research (Brussels, Belgium), acknowledges the Fund's financial support. The authors also acknowledge partial support by the American Jersey Cattle Association (Reynoldsburg, Ohio).

\section{REFERENCES}

Ducrocq, V., and B. Besbes. 1993. Solution of multiple trait animal models with missing data on some traits. J. Anim. Breed. Genet. 110:81-92.

Gengler, N., T. Dusseldorf, G. R. Wiggans, J. R. Wright, and T. Druet. 2001a. Heterogeneity of (co)variance components for Jersey type traits. J. Dairy Sci. 84:1772. Online. Available: http://www. adsa.org/jds/papers/0385/JDS0385.html.

Gengler, N., T. Dusseldorf, G. R. Wiggans, J. R. Wright, and T. Druet. 2001b. Joint estimation of variances and effects in the US Jersey type evaluation system. Interbull Bull. 26:34-40. Available: http://www-interbull.slu.se/bulletins/bulletin26/Gengler.pdf.

Gengler, N., G. R. Wiggans, and J. R. Wright. 1999. Animal model genetic evaluation of type traits for five dairy cattle breeds. J. Dairy Sci. 82:1350. Online. Available: http://www.adsa.org/ manuscripts/jds8311.

Gengler, N., G. R. Wiggans, J. R. Wright, and T. Druet. 2001c. Simultaneous accounting for heterogeneity of (co)variance components in genetic evaluation of type traits. J. Dairy Sci. 84 (Suppl. 1):247. Online. Available: http://www.adsa.org/jointabs/iaafs110.pdf.

Gengler, N., G. R. Wiggans, J. R. Wright, H. D. Norman, and C. W. Wolfe. 1997. Estimation of (co)variance components for Jersey type traits using a repeatability model. J. Dairy Sci. 80:18011806. 